\title{
Aspectos y avances en ciencia, tecnología e innovación
}

\author{
Marco A. Vega \\ Universidad Santo Tomás, Iquique, Chile. E-mail: mvegap@santotomas.cl
}

\begin{abstract}
Resumen: Este trabajo revisa y aplica algunos conceptos y relaciones entre conocimiento, investigación y desarrollo, y posteriormente, entre ciencia, tecnología, innovación y sociedad. Constituye una aproximación general, donde el conocimiento es visto como un proceso integrado que se inicia en el asombro y la curiosidad, se construye a través de la investigación y crece en múltiples disciplinas científicas básicas y aplicadas, que innovan y avanzan hacia el desarrollo y bienestar social, cultural y económico. A la luz de ciertos inventos y descubrimientos, nombres y figuras notables del saber, se discuten aspectos, avances y perspectivas de una cultura basada en el conocimiento, la información y la globalización. ciedad.

Palabras clave: Investigación, Ciencia, Tecnología, Innovación, So-
\end{abstract}

\section{Aspects and advances in science, technology and innovation}

\begin{abstract}
This work checks and applies some concepts and relations between knowledge, research and development, and later, between science, technology, innovation and society. It constitutes a general approximation, where knowledge is seen as an integrated process that begins with the amazement and curiosity, is built through investigation and grows in multiple basic and applied scientific disciplines, which innovate and advance towards development and social, cultural and economic well-being. In the light of certain inventions and discoveries, and of names and notable figures of knowledge, are discussed aspects, advances and perspectives of a culture based on knowledge, information and globalization.
\end{abstract}

Key words: Research, Science, Technology, Innovation, Society.

\section{Aspectos e avanços na ciência, tecnologia e inovação}

Resumo: Este trabalho verifica e aplica alguns conceitos e relações entre conhecimento, pesquisa e desenvolvimento, e, mais tarde, entre ciência, tecnologia, inovação e companhia. Constitui uma aproximação geral, onde o conhecimento é visto como um processo integrado que começa no assombro ea curiosidade, é construída através da investigação e cresce em várias disciplinas científicas básicas e aplicadas, que inovam e avançam para o desenvolvimento e bem-estar social, econômico e cultural. À luz de certas invenções e descobertas, nomes e figuras notáveis __do saber, são discutidos aspectos, avanços e perspectivas de uma cultura com base no conhecimento, a informação ea globalização.

Palavras-chave: Pesquisa, Ciência, Tecnologia, Inovação, Sociedade. 


\section{Conocimiento. Aproximación conceptual}

La historia del mundo y el desarrollo del ser humano, está ligado a los inventos y descubrimientos realizados a través de las épocas. Inicialmente el hombre se vio en la urgencia de resolver las necesidad más primarias, fue creando, uno tras otra, las herramientas y mecanismos que le permitieron transformar la realidad y comenzar un proceso civilizador que no ha cesado en su evolución (Alvarez 1990: 3). Desde el descubrimiento del fuego, invención de herramientas y desarrollo el lenguaje, hasta los más recientes y sofisticados descubrimientos que han permitido la desintegración del átomo y el uso de energía atómica, la exploración del cosmos y el desarrollo de múltiples disciplinas o campos del saber, como la biotecnología, nanotecnología e infotecnología (Yankovic 2010: 49), el ser humano, sujeto pensante, dotado de inteligencia, memoria y voluntad, ha realizado numerosos aportes, a través de figuras notables de las ciencias, artes y técnicas.

A lo largo de este camino, ambos términos, invento y descubrimiento, han estado estrechamente relacionados con el desarrollo y el progreso. Para el físico inglés, Roberto Hooke, el microscopio inventado en 1590 por Zacarías Janssen (holandés), y posteriormente perfeccionado por Galileo Galilei y Anton Van Leeuwenhoek, constituyó una herramienta fundamental para el descubrimiento de la célula en 1665. Mientras, que los grandes aportes de Galileo Galilei (1564 - 1642) a cerca de la composición de la Vía Láctea, los anillos de Saturno, los satélites de Júpiter, las manchas solares y la validez de la doctrina heliocéntrica de Copérnico, no hubieran sido posible sin el uso del telescopio inventado en 1608 por Hans Lippershey (óptico holandés), y posteriormente mejorado por el mismo. Por otra parte, las aplicaciones del rayo láser, inventado por Theo Maiman en 1960, no serían posible, sin el descubrimiento del efecto fotoeléctrico por Albert Einstein 1917. Tampoco, los sucesivos descubrimientos en la concepción del átomo que se ha tenido a lo largo de la historia, sin los aportes de John Dalton (1808), Joseph Thomson (1897), Ernest Rutherford (1911) y Niels Bohr (1913). O bien, una explicación satisfactoria a los factores de la herencia de Mendel, sin el descubrimiento de la estructura de la molécula de ADN, por los bioquímicos Watson y Cricks, en 1953. Y recientemente, el impacto de las técnicas de neuroimagen funcional en el desarrollo de las neurociencias (Logatt y Castro 2011: 36).

En la invención y el descubrimiento, el asombro, que experimentamos todos los seres humanos en muchos momentos de nuestra vida y que "constituye la semilla del conocimiento, o más dramáticamente expresado, el fulminante de todo el saber" (Rivera 1988: 24), se transforma en algo que sucede permanentemente. En su afán de entender y comprender, el ser humano se resiste a quedar sin ninguna explicación de las cosas, y adelanta ideas que sirven para encontrar un sentido a lo que ocurre (Sabino 1996: 125). Junto al asombro, esta la preparación. Santiago Ramón y Cajal (1999: 40), médico histólogo español, premio nobel de medicina y fisiología por sus descubrimientos sobre el sistema nervioso lo expresa muy bien "el des- 
cubrimiento no es fruto de ningún talento originariamente especial, sino del sentido común mejorado y robustecido por la educación técnica y por el hábito del meditar sobre los problemas. Así quien disponga de regular criterio para guiarse por la vida, lo tendrá también para marchar por el camino de la investigación.” En este contexto, Louis Pasteur, químico francés cuyos descubrimientos tuvieron una enorme importancia en el campo de las ciencias naturales, sobre todo en la química y microbiología, solía decir que "la casualidad favorece solo a las mentes preparadas" (www.profesorenline.cl/biografias/PasteurBiograf.htm).

El hecho es que ambos (inventos y descubrimientos) se originan a partir de diferentes tipos de conocimiento (práctico, estético, filosófico, científico, entre otros), diferentes fuentes de conocimiento (costumbres o tradiciones, autoridades o personas especializados, experiencias, ensayos y error, razonamiento lógico inductivo o deductivo e investigación científica o disciplinada, entre otras; Polit y Hungler 2000: 8) y diferentes pasos o métodos para obtenerlos, que se han traspasado de generación en generación, cambiando el conocimiento existente y afectando directa o indirectamente el bienestar económico, social y/o cultural. ${ }^{1}$

Este conocimientos o saber, obtenido de las facultades de la inteligencia, sobre la naturaleza, cualidades y relaciones de las cosas, cobra un significado adicional en el descubrimiento científico, porque nos entrega un pensamiento científico o método de trabajo, que se origina alrededor del siglo XV en los estudios de dos ilustres científicos: Galileo y Descartes (Logatt y Castro, 2011: 31), mediante la incorporación de experimentación en la comprobación de hipótesis, y posteriormente, la incorporación de la estadística al análisis de los resultados experimentales y que a su vez, puede ser validado o refutado a la luz de nuevas observaciones, es decir, nos muestra la aplicación de un método científico, una epistemología rigurosa (Cataldi 1998: 17), o bien flexibles y más abierto, que sin ser infalible, constituye un proceso, que en sus diferentes visiones o enfoques (positivistas, naturalista o fenomenológico, mixtos, entre otros; Hernández et al 2004), es muy válido y confiable en la obtención de conocimiento (Polit y Hungler 2000: 11). ${ }^{2}$ (Figura 1).

\section{Figura 1}

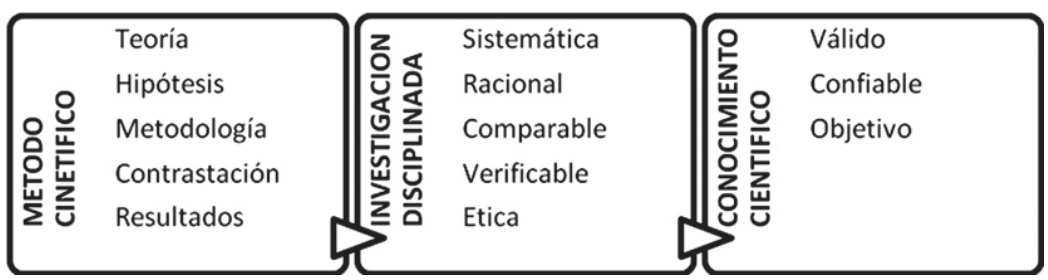

Fuente: a partir de Salkind 1997; Cataldi 1998; Rojas 2002; Hernández et al 2004. 
Este conocimiento científico, que no es absoluto e inmutable y está en constante revisión, se construye mediante la investigación científica, a partir de la identificación de un auténtico problema, y a los procesos de indagación sistemática, y contrastación de las teorías frente a los resultados, en un proceso social en el que intervienen, además del propio investigador, otros científicos que evalúan y aceptan o rechazan sus teorías, modelos, propuestas u observaciones (Campanario 2001a: 7). Mediante este proceso (Tabla 1), el volumen de conocimiento científico se a multiplicando aceleradamente (en la segunda mitad del siglo XX, se producía cada 15 ó 20 años una duplicación del volumen de conocimiento científico) (Díaz 2006: 117) y el hombre ha alcanzado una reconstrucción conceptual del mundo que es cada vez más amplia, profunda y exacta (Bunge 1960: 6). ${ }^{3}$

\section{Tabla 1. Investigación científica}

\begin{tabular}{|c|}
\hline Masa Crítica \\
\hline $\begin{array}{l}\text {-Profesional } \\
\text { - Investigador }\end{array}$ \\
\hline Formulación de Proyecto \\
\hline $\begin{array}{l}\text {-Idea y Problema } \\
\text { - Marco Teórico } \\
\text { - Hipótesis y Variables } \\
\text { - Metodologia } \\
\text { - Cronograma y Presupuesto }\end{array}$ \\
\hline Ejecución de Proyecto \\
\hline $\begin{array}{l}\text { - Validezy Confiabilidad de Instrumentos. } \\
\text { - Recolección de Información } \\
\text { - Análisis } \\
\text { - Confrontacióny Discusión de los resultados }\end{array}$ \\
\hline Comunicación de Resultados \\
\hline $\begin{array}{l}\text {-Publicación en Revista de corriente principal } \\
\text {-Ponencia en Encuentros de Especialidad. }\end{array}$ \\
\hline Transferencia de Resultados \\
\hline
\end{tabular}

Fuente: a partir de Ander-Egg y Aguilar 1996; Salkind 1997; Rojas 2002; Henríquez y Zepeda 2003; Contreras y Cumsille 2003; Hernández et al 2004.

\section{Investigación y desarrollo}

La investigación científica, inicialmente se cultivó de manera más individual y por sí misma (investigación básica). Sin considerar tanto las aplicaciones, más estas terminan llegando, a veces tardaban años, a veces siglos (Ramón y Cajal 1999: 37). Sin embargo, hoy en día, el concepto de investigación, que incluye desarrollo (I + D), integra, además, de la investigación básica, la investigación aplicada y el desarrollo experimental (OECD 2002: 30), que van desde la obtención de conocimiento hasta su aplicación y utilización. Es decir, la investigación científica, pasó de ser 
una labor de producción de conocimiento individual a una labor de tipo colectiva, desarrollada en redes o nexos nacionales e internacionales de equipos multidisciplinarios de investigadores(ras), que habitualmente operan, sobre problemas o cuestiones medianamente comprendidas, mal o aun no comprendidas, que tengan impacto social o económicas, entre otros, y sean innovadoras y competitivas, en líneas de investigación, desarrolladas en o entre universidades y centro de investigación especializados, con financiamientos internos, externos, públicos y privados. ${ }^{4}$ En ocasiones, perdiendo su carácter público, cada vez que la circulación del conocimiento científico, queda supeditada a las restricciones o condiciones del gran mercado, como en las patentes o en el secreto industrial (Rivera 1998: 4). Este gran cambio apunta, a que ahora a la producción de conocimiento se le exige con mayor fuerza resultados explícitos en su aplicación -innovación tecnológica y resolución de problemas sociales- lo que requiere compatibilizar intereses entre quienes generan este conocimiento y sus usuarios, exigiéndose entonces un cambio importante en los contenidos, participantes e interacciones entre todos ellos (Corvera y Loiseau 2004: 2).

La masa crítica que de manera disciplinada, abastecen el conocimiento actual, a través de la investigación científica, a su vez constituye un recurso humano especializado, con formación profesional demostrable (suficiencia investigadora), habilidades y destrezas (Tabla 2), y que son evaluados en la excelencia, creatividad y trascendencia de su obra, tanto en el desarrollo de su disciplina como en sus aportes a la culturización, que según Jaksic (2004: 1), debiera: generar y diseminar conocimiento original (hacer ciencia), enseñar (impartir docencia formativa a futuros científicos), tutorar (formar nuevos científicos de reemplazo), liderar y administrar (en beneficio de otros científicos), servir (a comunidad científica formada y en formación), divulgar (a la comunidad nacional que sustenta a los científicos) y participar (ser parte activa de sociedades científicas).

\section{Tabla 2. Capacidad de Investigación}

\begin{tabular}{ll}
\hline \multicolumn{1}{c}{ Destrezas } & \multicolumn{1}{c}{ Habilidades } \\
\hline $\begin{array}{l}\text { Manejo de campos del } \\
\text { conocimiento }\end{array}$ & $\begin{array}{l}\text { Búsqueda, selección y sistematización de información bibliográfica. } \\
\text { Interpretación y uso de la teoría }\end{array}$ \\
$\begin{array}{l}\text { Manejo de procedimientos, } \\
\text { técnicas y herramientas para } \\
\text { la obtención y el análisis de } \\
\text { información }\end{array}$ & $\begin{array}{l}\text { Instrumentales y metodológicas para el diseño y la aplicación de estos } \\
\text { Actitudes favorables para las }\end{array}$ \\
$\begin{array}{l}\text { investigación } \\
\text { Esta característica se refiere a un elemento afectivo indispensable, } \\
\text { desde la perspectiva en que asume la discusión. Las actitudes favorables } \\
\text { son: disciplina y rigor académico, curiosidad, laboriosidad, exigencia, } \\
\text { crítica y autocrítica; hábitos de trabajo intenso y regular; disposiciones } \\
\text { favorables para el trabajo en equipo, entre otros (Martinez, 1999). }\end{array}$ \\
\hline
\end{tabular}

Fuente: Martínez 1999; Weiss 2002; Moreno 2002. 
En este contexto, la investigación científica pasó a tomar un rol fundamental en el desarrollo, como portadora de un cambio que exige un verdadero conocimiento de la situación real, del estudio y de la teoría que lo sustenta (Díaz 2006: 13). Como una forma de documentar, identificar, ampliar y actualizar el conjunto de conocimiento. Como un bien público, que es socialmente benéfico (Benavente 2005: 64), para mejorar nuestra calidad de vida en los más variados aspectos. Y como un factor esencial para la adopción y promoción de políticas públicas y privadas de cualquier sociedad avanzada. Que a su vez, resulta determinante, para los países en vías de desarrollo, puesto que permite (Lederman y Maloney 2004: 1) identificar adaptaciones o aplicaciones comerciales de este conocimiento que son viables. Disponer de profesionales familiarizados con las fronteras del conocimiento que impartan capacitación. Evitar depender exclusivamente de lo que genera los países más avanzados. Y de acuerdo a las nuevas teorías de crecimiento económico, generar (en una unidad productiva) la incorporación de productos y procesos inexistentes hasta entonces, con retornos económicos crecientes (Benavente 2005: 53).

\section{Ciencia, tecnología, innovación y sociedad}

Desde una aproximación conceptual, la ciencia, ya sea formal o fáctica, se caracteriza por un conocimiento racional, verificable y por consiguiente falible (Rojas, 2002: 9), aceptado, sistematizado y validado por la comunidad científica (Raviolo et al, 2010: 30), y como actividad (investigación), en cuanto se aplica al mejoramiento de nuestro medio natural y artificial, y a la invención y manufactura de bienes materiales y culturales, se convierte en tecnología (Bunge 1960: 6). Así, la investigación científica se distingue de la tecnológica en que su motivación es satisfacer la curiosidad y comprender el mundo, carece de una orientación estratégica, genera nuevo conocimiento, su producto fundamental son las publicaciones, utiliza el método científico y su validez es universal (Feller, 2011: 8). UNESCO (1988: 1), propone 24 campos de disciplinas, las cuales incluyen diversas subdisciplinas, y últimamente, el manual de Frascati, elaborado por la OECD, distingue 42 campos de la ciencia y la tecnología, en seis grandes ramas: ciencias agrícolas, ciencias naturales, ciencias sociales, ciencias médicas, humanidades e ingeniería y tecnología.

La actividad técnica ha precedido en milenos a la ciencia, como se aprecia en los inventos, por lo que no constituye únicamente una mera aplicación de conocimientos científicos (UNESCO 2005a: 32). La Innovación en cambio, tecnológica o no, constituye la implementación de un producto (bien o servicio) o proceso nuevo o con un alto grado de mejora, o un método de comercialización u organización nuevo aplicado a las prácticas de negocio, al lugar de trabajo o a las relaciones externas (Sánchez y Castrillo 2006: 5).

En el desarrollo de la humanidad, la Innovación, tiene un despegue más temprano en comparación a la Ciencia y la Tecnología, y a partir de 
esta en el marco de una sociedad que crece y avanza aceleradamente en información, comunicación y globalización, donde ya varios países latinoamericanos, han elaborado lineamientos y estrategias en innovación y competitividad nacional o global, económica y cultural, para la inserción y progreso en diferentes ámbitos internacionales. La Ciencia y la Tecnología, en cambio, despega a comienzos del siglo XVIII (antes habían iniciativas importantes, pero más focalizadas y menos conocidas), con la llegada de la Revolución Industrial, al detonarse una explosión de aplicaciones científicas y tecnológicas que, desde entonces se han multiplicado aceleradamente sin pausa, impactando o beneficiando en diversos campos (Boisier 2010: 21). ${ }^{5}$ En América Latina, la Ciencia y Tecnología, cobran importancia significativa a partir de la Segunda Guerra Mundial, entre las décadas de 1950 a 1980, con el establecimiento de la Organización de las Naciones Unidas, en particular UNESCO, que dio un impulso a la ciencia en los países de menor desarrollo relativo, los cuales posteriormente crean organismos estatales de ciencia y tecnología, para establecer sus políticas nacional y canalizar recursos para fomentar la investigación (Rivera 1998: 6). No sin antes encontrar diversos obstáculos en su expansión producto de cambios súbitos o forzosos de regímenes políticos y estancamiento económico (ídem).

En el marco económico y social, algunos aspectos propiciaron un entorno que facilitó las actividades de ciencia, tecnología e innovación en el país. La creación de las principales instituciones orientadas a promover el desarrollo y la implementación de la Investigación y Desarrollo en Chile, como es la Corporación de Fomento de la Producción (CORFO), cuya misión es fomentar el emprendimiento y la innovación para mejorar la productividad de Chile y alcanzar posiciones de liderazgo mundial en materia de competitividad y la Comisión Nacional de Investigación Científica y Tecnológica de Chile (CONICYT), que se orienta al fomento de la formación de capital humano y al fortalecimiento de la base científica y tecnológica del país. Por otra parte, la apertura económica de Chile, que en un principio provoco crisis en muchas empresas, acabó produciendo la reconversión en parte importante del sistema productivo, desarrollando un grupo empresariado orientado a la competitividad internacional, que comienza a demandar tecnología igual o superior a sus competidores, con un flujo considerable de transferencia tecnológica (como equipamiento, infraestructura, servicios o mano de obra calificada) desde empresas internacionales de punta (sobre todo en recursos naturales y servicios), y más tarde, comienza a demandar innovación, para diferenciarse de sus competidores en la oferta de productos y servicios (Yutronic 2004: 116). Se sucede la privatización temprana de mayoría de empresas estatales y la desregulación de muchos sectores de la economía, como una forma de promover la iniciativa emprendedora y la competencia (ídem). En este escenario, la capacidad de generar y absorber innovación (crear y comercializar tecnología), supuso y supone una base sólida en investigación básica, que hasta la década de los 90 en Chile, estaba fuertemente impulsada por el Fondo Nacional de Desarrollo Científico y Tecnológico (FONDECYT), pero como no se traducían en aplicaciones prácticas o en innovación tecnológicas, a través de políticas públicas surgen los Fondos Tecnológicos (FONDEF, FONTEC, 
FDI, etc.), para incrementar el esfuerzo nacional en innovación a través del fortalecimiento de la demanda tecnológica, impulsando una mayor participación de la empresa privada en este esfuerzo (Corvera y Loiseau 2004: 24), y potenciando el vínculo universidad o centros de investigación (generación-transmisión de conocimiento) y empresa. Otro impulso, se produce a través de la Comisión Nacional de Innovación y Competitividad, que en su Agenda 2010, circunscribe el desarrollo del país en pasar desde una economía de materias primas basadas en recursos naturales, a una basada en innovación, capital humano de calidad y conocimiento, y que en el marco de sus siete líneas estratégicas y desafíos, reconoce la importancia fundamental del capital humano de calidad (Villarroel 2010a: 18), la ciencia base orientada estratégicamente (Villarroel 2010b: 5) y la innovación empresarial. Ejes que adquieren más significado, en los sectores de la economía chilena con alto potencial de desarrollo (acuicultura, alimentos procesados, fruticultura, minería del cobre, offsshoring, porcicultura-avicultura, servicios financieros, turismo) y en el contexto de una correcta identificación de los requerimientos científicos y tecnológicos (Rosas 2010: 7). A este escenario se suma el gasto en Ciencia y Tecnología en relación al PIB, estancado en la segunda mitad de los 90 (ídem), y que luego tuvo un crecimiento sin precedentes en el periodo 2005-2010 (Boisier 2010: 3), aunque sigue siendo de proporción baja, en comparación con los países de América del Norte (Rivera 1998: 10). Y el hecho, que la mitad de las diferencias observadas en el ingreso per cápita y el crecimiento del PIB de los países se debe a disparidades en la Productividad Total de los factores (PTF), por lo general, relacionada con los avances tecnológicos e incluso, que gran parte de la creciente brecha que existe entre países ricos y pobres no se debe a diferencias en la inversión de capital, sino a su desarrollo en el área tecnológica (Lederman y Maloney 2004: 1). Se produce entonces, una orientación país en materia de institucionalidad, consolidando metas y estrategias en ciencia, tecnología e innovación y fortaleciendo sus avances, a través del incremento de estos y otros indicadores científicos y económicos como, el gasto en investigación y desarrollo para financiar mano de obra calificada e infraestructura necesaria, el capital humano altamente especializado (número graduados de doctorado país, por millón de habitantes), cantidad de publicaciones científicas en revistas internacionales (índice de citación: factor de impacto), fondos concursables, productos de la innovación (patentes recibidas por investigadores residentes país, por la población nacional), porcentaje de ventas que corresponde a productos nuevos, entre otros indicadores (Corvera y Loiseau 2004: 4; Benavente 2005: 54). ${ }^{6}$

A lo largo de este procesos, el vinculo (investigador-empresa), no queda exento de algunas barreras, como por ejemplo la inversión privada en I + D, que en la percepción de los investigadores, corresponde a la disposición de la empresa sólo a realizar inversiones que aseguren una rentabilidad a corto plazo y a su vez, que espera recibir estímulos que tengan relación con beneficios a nivel tributario, de obtención de patentes o retornos rápidos y efectivos (Ramos 2004: 52). También, se percibe aspectos relacionados con un desequilibrio entre investigación básica y aplicada, en 
términos de asignación desigual de fondos y en la complejidad o accesibilidad a los fondos. Además, de una percepción de un darwinismo social, en donde sobrevivan los que más capacidades tienen y que logren mayor competitividad, en parte, a raíz de un proceso de globalización que no está generando un incremento uniforme de progreso y desarrollo en todas las regiones del mundo (Rivera 1998: 8).

A esta altura, se evidencia, que la capacidad de innovación de un país es el resultado de un entorno macroeconómico e institucional adecuado, en conjunto con el funcionamiento del sistema educativo, productivo y de ciencia y tecnología; de las interrelaciones entre todos ellos y de su interacción con el sistema social (Corvera y Loiseau 2004: 2). Donde la institucionalidad, alfabetización científica y difusión, jueguen un rol preponderante en el crecimiento, articulación y proyección de la Ciencia, Tecnología, Innovación en la Sociedad (Chile-Ciencia 2000: 5).

En materia de educación o alfabetización científica tecnológica, definida como el conocimiento de los conceptos básicos a cerca de la ciencia y la técnica, de una comprensión de cómo la ciencia funciona (Barros et al 2005: 2), del desarrollo de una cultura científica, que no sólo permita la formación de especialistas en ciencias y tecnología, sino que desde el punto de vista democrático, permite a los ciudadanos participar en las decisiones que las sociedades deben adoptar en torno a problemas científicos y tecnológicos cada vez más complejos (UNESCO 2005a: 19), la educación en ciencias está viviendo un proceso de acomodación. Hay un porcentaje de nuestra población que se encuentra ligeramente satisfecha y la está cambiando y mejorando. Más allá de la absoluta idoneidad de los indicadores nacionales e internacionales en educación (Santander 2006: 111), los resultados, no son lo más esperados, nos son altos respecto de los estándares internacionales, ni uniformes respecto del estrato socioeconómico y geográfico; se destinan pocos recursos, es un sector no bien remunerado y faltan espacios para la reflexión y el perfeccionamiento. De acuerdo a Calvo y Elizalde (2009: 3), el fracaso escolar tiene su origen epistemológico en la concepción de enseñanza y aprendizaje que sustenta la escuela y no en el alumno y profesor, ni en la adecuación o pertinencia de los programas de estudio. En otras palabras a la obsesión por la respuesta, antes que por la pregunta, con lo que inhibe sino aniquila, la curiosidad y la propensión a aprender lúdicamente y la búsqueda de lo nuevo, de lo posible. Ya en el siglo pasado, Albert Einstein, decía que "es casi un milagro que los métodos modernos de educación no hayan estrangulado completamente la bendita curiosidad de investigar" (Yankovic 2010: 54). La crítica más importante a la educación que reciben los niños y jóvenes chilenos en esta área son (Feller 2011: 48): Se presenta a la Ciencia y tecnología como un cuerpo de conocimientos, no como una actividad que persigue el descubrimiento del mundo basado en la curiosidad y creatividad, actitudes inherentes a toda persona; se privilegia la memorización en una gran cantidad de contenidos, antes que el aprendizaje del método y el pensamiento científico, a través de la práctica y exploración del entorno; las instituciones de educación superior que imparten carreras de Pedagogía se encuentran totalmente 
separadas de las instituciones que concentran la actividad científica y tecnológica, perjudicando la formación de los profesores en estas materias.

En el marco de los Profesores y Docentes de Ciencia, parece necesario seguir incursionando en políticas públicas que mejoren su formación y desempeño (Barros et al 2005: 10). Analizar experiencias exitosas en materia de educación y ampliar y diversificar los modelos de enseñanza (Schiefelbein 1998: 14; Schiefelbein y Zúñiga 2002: 1; González y Rasilla, 2011:15). No centrarse únicamente en la enseñanza, sino también en la investigación e involucrar investigadores activos en materia de educación (Schiefelbein 2008: 31). Y a su vez, evitar la educación científica centrada en la mera transmisión de conocimiento y en los estereotipos socialmente aceptado (UNESCO 2005a: 31): como descontextualizada de la sociedad y el ambiente, carácter individualista y elitista, concepción empiro-inductivista y ateórica, carácter rígida, algorítmica e infalible, visión aproblemática y ahistórica, carácter exclusivamente libresco, visión exclusivamente analítica y acumulativa de crecimiento lineal.

A este respecto, la didáctica de las ciencias, cuyo objeto de estudio son los sistemas de enseñanza-aprendizaje que abordan fenómenos naturales y materiales, a través de dos dimensiones, el análisis de problemas y dificultades de aprendizaje y la búsqueda y experimentación de nuevos enfoques de enseñanza (Campanario 2001b: 12), constituye otra herramienta de importancia fundamental, cuya investigación está mostrando que la comprensión significativa de los conceptos exige superar el reduccionismo conceptual y plantear el aprendizaje de las ciencias, como una actividad, próxima a la investigación científica, que integre los aspectos conceptuales, procedimentales y actitudinales (UNESCO 2005a: 26) . Otra herramienta es la educación informal, que ya tiene un rol fundamental en la formación de futuros profesional y científica (Barros 2005: 4), que como procesos informales caóticos, emergentes y autoorganizados, poseen una complejidad y un nivel de abstracción muy alto, que supera sin problemas a los de la escuela (Calvo y Elizalde 2009: 3). Además, de otros procesos que están integrando metodología y tecnología e innovando en la educación de las ciencias.

Se hace necesario, fortalecer simultáneamente la educación e investigación científica, potenciando todas las capacidades de investigación (básica, aplicada, tecnológica), para construir una sociedad en la cual todos los individuos puedan llegar a ser personas, con una vida digna y honrosa (Rivera 1998: 17). Fomentar la divulgación en materia de ciencia y tecnología, como fue indicado recientemente por las Sociedades Científicas (Organizaciones Científicas 2010), en el marco de la catástrofe del sur de Chile el día 24 de febrero de 2010 y en virtud de algunos estudios científicos que dejaban notoria y anticipada evidencia y prevención (Ruegg et al 2009: 78; Cecioni y Pineda 2009: 379), en la sociedad del conocimiento es fundamental la información científica oportuna, los espacios públicos de reflexión y el fortalecimiento de los vínculos entre la comunidad científica nacional y las autoridades del país, responsables de las decisiones. Tomar 
conciencia sobre el valor real de la ciencia y tecnología para el desarrollo país, incrementando la divulgación, participación y disposición hacia la ciencia y tecnología, tal que no se produzca, por ejemplo, falta de accesibilidad, especialmente en protagonistas del quehacer nacional (Feller, 2011: 8).Y seguir legislando e impulsando talleres y comités de ética y bioética (UNESCO 2005b: 3; Arias et al. 2010: R-27), en el marco del crecimiento y posibilidades de la ciencia, tecnología e innovación, para mejorar la protección del entorno y sus integrantes, reducir problemas o eventos adversos (como la contaminación, falta de conservación y uso sustentable de la diversidad biológica, desigualdades económicas y sociales, empoderamiento bélico, etc.), y prevenir otros. 


\section{Notas}

${ }^{1}$ Tipos de Conocimientos. a) Práctico: Arquímides (siglo III AC, en inventos como la rueda dentada, órgano hidráulico, poleas, palancas, etc.) y de Johannes Gutenberg (inventor de la imprenta de tipos móviles, que significo una revolución cultural). b) Artístico y/o estético: surrealismo de Salvador Dalí, que revolucionaron la pintura junto con Picasso y Miro en el siglo XX; las letras de Gabriela Mistral y Pablo Neruda, premios nobel de literatura. c) Filosófico: Sócrates, Aristóteles, Platón, Santo Tomás de Aquino, René Descartes e Immanuel Kant, entre muchos otros. d) Científico: cuidadosa y rigurosamente realizo, excesivamente racional (empleo y predominio de la razón para la explicación de los fenómenos), sistemático (unificar conocimiento fundado, ordenado y coherente) y verificable (por la observación o experimentación), cuyo propósito está en la comprensión y control de los fenómenos (Rojas 2002: 9), evidenciado en los aportes de Hipócrates, médico griego 400 años AC, quién es considerado el padre de la medicina alopática, Isaac Newton, notable científico y matemático inglés del siglo XVII, Antoine Lavoisier, químico francés del siglo XVIII, considerado el padre de la química moderna, Roberto Koch y Louis Pasteur, siglo XIX, considerados los padres de bacteriología y Albert Einstein, físico del siglo XX, quien con sus ideas cambió la concepción del mundo.

${ }^{2}$ Métodos Científicos. a) El riguroso diseño experimental con arvejas cultivadas en el jardín de su monasterio, a lo largo de varios años, por el sacerdote y botánico Gregor Mendel, que en el año 1866 dio origen a las leyes que rigen el mecanismo hereditario en los seres vivos. b) El meticuloso y minuciosos trabajo longitudinal, de tipo exploratorio-descriptivo y alcance correlacional-explicativo que en el año de 1859 Charles Darwin, naturalista inglés, presento en un cuerpo coherente de observaciones que explicaron el concepto de evolución biológica en su libro el origen de las especies por selección natural, estableciendo las bases de la teoría de la Evolución.

${ }^{3}$ Multiplicación acelerada del conocimiento. a) Desarrollo de las teorías del aprendizaje conductistas (1886-1950; Pavlov, Watson y Skinner), cognitivistas (1900-1950; Wertheimer, Köhler, Koffka, Gagne, Ausubel) y constructivismo (1920-actual; Piaget, Vigotsky, Bruner). b) Progreso de enfermedades incurables, desde que en 1981 el médico Michael Gottieb reporta los primeros casos de una enfermedad nueva, el SIDA, dos años más tarde, el investigador Luc Montagnier del Instituto Posteur de París descubre el virus, y hoy en día, a 30 años, se reportan nuevos avances. c) En salud, prácticamente no existe un mes en el que no se publique algún informe para alabar o no las propiedades de la cafeína, en torno al café (Centro para la Ciencia del Interés Público, CSPI: www.diariosalud.net). d) En el campo de la biotecnología, sólo en 16 años se paso de intentos por clonar ratones en la Universidad de Harvard, Estados Unidos, al primer organismo animal obtenido por clonación, la oveja Dolly. e) En el área de las comunicaciones, en 1969 el departamento de defensa de los Estados Unidos crea el sistema ARPANet, embrión de internet, y ya en 1993, Internet se convierte en el medio de información más usado en el mundo (Yankovic 2010: 47).

${ }^{4}$ Investigaciones Científicas. a) El Proyecto Genoma Humano (PGH), un acontecimiento del siglo XX, que comenzó el año 1990 en un consorcio público de 19 centros de investigación de 6 países, cuyo objetivo fue secuenciar y ordenar más de 3000 millones de nucleótidos que componen el genoma humano, identificar en ella los genes que existen, almacenar esta información en bases de datos públicas, desarrollar tecnologías y herramientas para el análisis de estos datos y establecer las cuestiones éticas, legales y sociales que resultan de dicha investigación (Yankovic 2010: 48). b) El Grupo de Trabajo de Biodiversidad Acuática, creado en 1999 por el Comité Oceanográfico Nacional (CONA, Chile), integrado por inves- 
tigadores de universidades y profesionales de instituciones públicas, cuyo objetivo ha sido incentivar el estudio de la Biodiversidad Acuática, fomentando el desarrollo de la taxonomía, sistemática y biogeografía, cuyos resultados se han materializado en publicaciones ininterrumpidas y en el año internacional de Biodiversidad (Bicentenario de Chile), en un libro indispensables que reúne 37 artículos referidos a diversos de los grupos vegetales y animales que habitan las aguas continentales y marinas del país (Palma et al 2010: 4).

${ }^{5}$ Impacto de la Ciencia y Tecnología (Boisier 2010: 25). a) Científico, en el desarrollo de la ciencia misma, avance en el conocimiento, nuevas especialidades o disciplinas, nuevos investigadores e investigaciones, etc. b) Tecnológico, en nuevas tecnologías disponibles, innovación de productos, servicios o procesos. c) Económico, en mejoras económica, nuevas fuentes financiamiento, inversión, actividades productivas o mercados. d) Social, en el mejoramiento en el bienestar, conductas, prácticas y actividades de personas y grupos. e) Cultural, en el enriquecimiento del conocimiento y la comprensión de la realidad por los individuos de una sociedad. f) Político, en la elaboración de estándares, normas, leyes o políticas públicas y modificación de interés o actitudes. g) Educacional, en los currículos o programas de estudio, herramientas pedagógicas, competencias, etc. h) Salud, en la mejora del tratamientos de enfermedades o en el sistemas de salud. i) Ambiental, en el mejoramiento en el manejo o mejoramiento del entorno ambiental. j) Organizacional, en la planificación, organización del trabajo, administración, recursos humanos. k) Simbólico, en el aporta prestigio, credibilidad o interés a una empresa, organización o país.

${ }^{6}$ Fuentes Indicadores Ciencia y Tecnología. a) Internacionales: Red Iberoamericana de Indicadores de Ciencia y Tecnología (RICYT), la Organización para la Cooperación y Desarrollo Económico (OECD), el Institute for Scientific Information (ISI), el Banco Mundial, el Observatorio de Ciencia, Tecnología e Información (KAWAX), etc.). b) Nacionales: Comisión Nacional de Investigación Científica y Tecnológica (CONICYT), el Ministerio de Economía, Fomento y Reconstrucción (DPI), el Consejo de Rectores de Universidades Chilenas (CRUCH), la Academia Chilena de Ciencias, Consejo Nacional para la Innovación y Competitividad (CNIC, Chile), Universidades Privadas, revistas temáticas, entre muchas otras. 


\section{Bibliografía}

Alvarez del Real, M.A. (1990), Inventos y descubrimientos más famosos. Ed. América S.A. Panamá. 288 págs.

Ander-Egg, E. y M.J. Aguilar I. (1996), Como elaborar un proyecto. Guía para diseñar proyectos sociales y culturales. Ed. LUMEN/HVMANITAS. Buenos Aires. 127 págs.

Arias, J.L., R. Bull, N. Giuliani, M. Santos, T. Ureta y C. Valenzuela (2010), Funcionamiento de Comitésde Bioética Institucionales. Biological Research. Vol. 43(Suplemento A). R-27.

Barros, F., G. Pino, P. Felmer, R. Gonzalo y E. Tirapegui. (2005), “Ciencia y Sociedad”. 1-20 págs. En J.E. Allende, J. Babul, S. Martínez y T. Ureta. Análisis y Proyección de la Ciencia Chilena 2005. Academia Chilena de Ciencias. 427 págs.

Benavente, J.M. (2005), “Innovación tecnológica en Chile: dónde estamos y que se puede hacer”. Economía Chilena Vol. 8 Nº 1.

Boisier, P., M.E. (2010), “Ciencia y la Tecnología en Chile: ¿Para Que?”. Comisión Nacional de Investigación Científica y Tecnológica (CONICYT). Gobierno de Chile. 132 págs. www.conicyt.cl.

Bunge, M. (1960), La Ciencia. Su método y su filosofía. Eudeba. Buenos Aires.

Calvo, C. y A. Elizalde (2009), “Educación: creación de nuevas relaciones posibles”. Polis $N^{o}$ 25, Revista de la Universidad Bolivariana, Santiago. http://polis.revues.org/292

Campanario, J.M. (2001-2002ª), Como escribir y publicar un artículo científico. Cómo estudiar y aumentar su impacto. Universidad de Alcalá. Ministerio de Educación, Cultura y Deporte. Madrid (España). www.uah.es/ otrosweb/jmc.

Ídem (2001-2002 ), La enseñanza de las ciencias. Preguntas y respuestas. Universidad de Alcalá. Ministerio de Educación, Cultura y Deporte. Madrid (España). www.uah.es/otrosweb/jmc.

Cataldi, A., R.M. (1998), Los informes científicos (Como elaborar Tesis, Monografías, Artículos para publicar, etcétera). Ed. Lugar Editorial. Buenos Aires. 95 págs.

Cecioni, A. y Pineda, V. (2009), “Geology and Geomorphology of Natural Hazards and Human-Induced Disasters in Chile”. Developments in Earth Surface Processes, 13: 379-413 págs. 
CNIC (2010), “Agenda de Innovación y Competitividad Chile 2010 2020”. Consejo Nacional de Innovación para la Competitividad (CNIC) y Secretaría Ejecutiva. http://biblioteca.cnic.cl/content/view/751136/Agenda-Innovacion-y-Competitividad-2010-2020.html\#content-top.

Contreras, T., G. y F. Cumsille G. (2003), “Metodología de la Investigación Científica”. Bol. Cient. Asoc. Segur. 5(9): 29-32 págs.

CONICYT (2007), “Antecedentes CONICYT. Estrategia 2006 - 2010”. Departamento de Estudios y Planificación estratégica. Comisión Nacional de Investigación, Ciencia y tecnología. Abril 2007. Gobierno de Santiago de Chile.

Corvera, M.T. y V. Loiseau. (2004), “Ciencia y tecnología, Indicadores de la situación chilena”. Biblioteca del Congreso Nacional de Chile. Depto. Estudios, Extensión y publicaciones. DEPESEX/BCN/SERIE ESTUDIOS/ AÑO XIV, N 14 .

Díaz, V.P, N. (2006), Metodología de Investigación Científica y Bioestadística. Universidad Finis Terrae. Ril Editores. Santiago de Chile. 328 págs.

Feller V., J.C. 2011. "El sistema chileno de fomento a la ciencia y tecnología: una evaluación desde la óptica de distintos protagonistas del quehacer nacional”. Informe Final. Katalejo. Sociedad de Biología de Chile. 59 p.

González, L.M. y M. Rasilla. 2011. Una estrategia para el aprendizaje de la cultura científica. Formación Universitaria. 4(2): 15 - 26.

Henríquez, E., F. y M.I. Zepéda G. (2003), “Preparación de un Proyecto de Investigación”. Ciencia y Enfermería. IX (2): 23-28 págs.

Hernández, R., C. Fernández C. y P. Baptista L. (2004), Metodología de la Investigación. McGraw-Hill Interamericana. 705 págs.

Jaksic, F. (2004), “Sobre el premio nacional de ciencias naturales”. Biol. Res. 37: 719-720 págs.

Jiménez, G., S.A. y J. Morales V. (2006), “La capacidad para la investigación en las trayectorias escolares de maestría”. Congreso Estatal de investigación Educativa. Actualidad, Prospectivas y Retos.

Lederman, D. y W.F. Maloney. (2004), “Innovación en Chile: ¿Dónde estamos?”. En foco, 18: 14. http://www.expansiva.cl/publicaciones/en_foco/ detalle.tpl?iddocumento $=05052004210007$

Logatt C., G.y M. Castro (2011), Neurosicoeducación para todos. Neurociencia para el cambio. Asociación Educar. 1era Edición. Argentina. 87 p. 
Martinez, F. (1999), “¿Es posible una formación sistemática para la investigación educativa?. Algunas reflexiones”. Revista Electrónica de Investigación Educativa, Ciudad de México. 1(1): 47-53 págs.

Moreno, M.G. (2002), “Hacia nuevas formas de concebir la formación para la investigación educativa”. Educar, Nº 21.

Organizaciones Científicas (2010), “Científicos unidos por la reconstrucción de Chile”. Facultad de Ciencias. Universidad de Chile. http:// www.ciencias.uchile.cl/ciencias/facultad_resena.php

OECD (2002), “Medición de las actividades Científicas y Tecnológicas”. Manual de Frascati. Organización para la Cooperación y Desarrollo Económico.

Palma, S., P. Báez y G. Pequeño. (2010), Bibliografía sobre Biodiversidad Acuática de Chile. Comité Oceanográfico Nacional - Chile. 459 págs.

Polit, D. y B.P. Hungler. (2000), Investigación científica en ciencias de la salud. McGraw-Hill Interamericana. 715 págs.

Ramón y Cajal, S. (1999), Reglas y consejos sobre investigación científica. Los tónicos de la voluntad. Consejo Superior de Investigaciones Científicas. Madrid (España). 203 págs.

Ramos, R., B. (2004), “Investigación y desarrollo: la percepción de investigadores y empresas. Ciencia, Tecnología e Innovación en Chile”. Comisión Nacional de Ciencias y Tecnología (CONICYT, Chile). www.conicyt.cl/ 573/articles-7506_estudio.pdf

Raviolo, A, P. Ramírez, E. A. López y A. Aguilar. 2010. Concepción sobre el conocimiento y los modelos científicos: Un estudio preliminar. Formación Universitaria. 3(5): 29 - 36.

Rivera, C., C. (1988), La aventura de la ciencia. Los primeros pasos de un joven científico. Ediciones Universidad Católica de Chile. Colección TELEDUC. Chile. 244 págs.

Rivera, M. (1998), “Ciencia, tecnología, educación e investigación: ¿elementos fundamentales para la competitividad global o para el desarrollo humano?”. Universidad del Turabo. Puerto Rico.

Rojas, C., M. (2002), Manual de Investigación y Redacción Científica. Lima. 82 págs.

Rosas, H., P. (2010), “Informe de Avance: Identificación de requerimientos Científicos y Tecnológicos derivados de sectores priorizados”. Consejo Nacional de Innovación para la Competitividad (CNIC). http:// biblioteca.cnic.cl/content/view/897984/Identificacion-de-requerimientos- 
cientifico-tecnologicos-derivados-de-cuatro-sectores-priorizados. html\#content-top.

Ruegg, J.C., A. Rudloff, C. Vigny, R. Madariaga, J.B. de Chabalier, J. Campos, E. Kausel, S. Barrientos y D. Dimitrov. (2009), "Interseismic strain accumulation measured by GPS in the seismic gap between Constitución and Concepción in Chile”. Physics of the Earth and Planetary Interiors, 175: 78-85 págs.

Sabino, C.A. (1996), Los caminos de la Ciencia. Una Introducción del Método Científico. Ed. Panapo. Caracas. 151 págs.

Salkind, N.J. (1997), Métodos de Investigación. Prentice Hall de Mexico. 380 págs.

Sánchez, M.A. y R. Castrillo. (2006), “La tercera edición del manual de Oslo: cambios e implicaciones. Una perspectiva de capital intelectual”. Revista $I+D$. № 35: 16 págs.

Santander, M. (2006), Ideas para la educación de calidad. Fundación Libertad y Desarrollo. 422 págs.

Schiefelbein, E. (1998), "Veinte modelos didácticos para América Latina”. En: Catálogo de modelos didácticos de Göttingen. Instituto para la Didáctica Intercultural. Universidad de Göttingen.

Schiefelbein, E. (2008), Desafíos para los investigadores en educación de América Latina. Magis, Revista Internacional de Investigación en Educación, $1,31-44$.

Schiefelbein, E y R. Zúñiga. (2002), “El Syllabus: Viviendo un aprendizaje autónomo”. Universidad Santo Tomás. Recuperado el 1 de septiembre, 2008, de http://www.ust.cl/link.cgi/Biblioteca/Publicaciones/Libros/1915

UNESCO (1988), "Proyecto de nomenclatura internacional normalizada relativa a la Ciencia y tecnología”. Organización Naciones Unidas para la Educación, la Ciencia y la Cultura.París.

UNESCO (2005ª), ¿Cómo promover el interés por la cultura científica?. Una propuesta didáctica fundamentada para la educación científica de jóvenes de 15 a 18 años. Oficina Regional de Educación de la UNESCO para América Latina y el Caribe OREALC/UNESCO Santiago.

Ídem (2005b), “Declaración Universal sobre Bioética y Derechos Humanos”. Organización de las Naciones Unidas para la Educación, la Ciencia y la Cultura.

Villarroel, G., K. (2010ª), “Avances, desafíos y propuestas en Capital Humano. Documento de Referencia”. Consejo Nacional de Innovación para 
la Competitividad (CNIC). http://biblioteca.cnic.cl/content/view/916114/ Avances-Desafios-y-Propuestas-en-Capital-Humano.html\#content-top.

Ídem (2010 b), “Avances, desafíos y propuestas en Ciencia”. Documento de Referencia. Consejo Nacional de Innovación para la Competitividad (CNIC). http://biblioteca.cnic.cl/content/view/915715/Avances-Desafiosy-Propuestas-en-Ciencia.html\#content-top.

Weiss, E. (2003), “Diagnósticos, panoramas y estados de conocimientos”. En Weiss, E. Coord. (2003). Estado del Conocimiento: El campo de la investigación educativa 1992 - 2002. COMIE de México.

Yankovic, B., N. (2010), Inventos y descubrimientos. Universidad de Talca. www.educativo.utalca.cl/medios/educativo/profesores/media/recursos/ inventos_descubrimientos.pdf

Yutronic, J. (2004), “Ciencia, Tecnología e Innovación en Chile a las puertas del siglo XXI”. Temas de Iberoamérica. Globalización, Ciencia y Tecnología. http://www.innovacion.cl/sites/default/files/yutronic_0.pdf

Recibido: 24.06.2011

Aceptado: 18.07.2012 